\title{
ReaR
}

ISNN 19894090

Revista electrónica de AnestesiaR

CASOS CLÍNICOS

Noviembre 2021

\section{Manejo anestésico de la Osteogénesis Imperfecta. Revisión de la bibliografía a propósito de un caso.}

Serrano de los Santos MD, Serrano de los Santo MJ

\section{H.U. Virgen del Rocío, Sevilla.}

\section{Resumen}

Los pacientes afectos de Osteogénesis Imperfecta son, por su patología, candidatos a requerir más de una intervención quirúrgica y por tanto, a ser anestesiados. Se realiza revisión en busca de las alteraciones fisiopatológicas asociadas a esta entidad que con más frecuencia se hallan en esta población, y sus potenciales implicaciones anestésicas.

\section{Introducción}

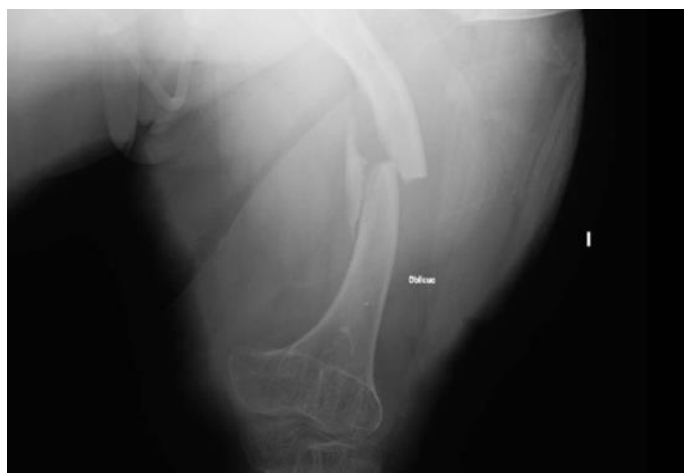

Los pacientes afectos de Osteogénesis Imperfecta son, por su patología, candidatos a requerir más de una intervención quirúrgica y por tanto, a ser anestesiados. Se realiza revisión en busca de las alteraciones fisiopatológicas asociadas a esta entidad que con más frecuencia se hallan en esta población, y sus potenciales implicaciones anestésicas.

La osteogénesis imperfecta (OI) o huesos de cristal fue definida por Vrolik en 1849, constituye un síndrome osteoporótico hereditario y tiene una incidencia de 1 por cada 20.000 nacimientos, con presentaciones graves en 1 de cada 50.000 nacidos.
Es causada por una alteración en la matriz proteica, que lleva a una formación anómala del hueso con rápido recambio. $\mathrm{Su}$ expresión clínica más frecuente son fracturas recurrentes después de traumatismos generalmente leves.

Clásicamente, esta enfermedad se ha descrito en las clasificaciones clínicas secundarias a la osteopenia generalizada; sin embargo, constituye una conectivopatía con repercusión sistémica debida a defectos cuantitativos o cualitativos en la síntesis de dicha proteína (quienes portan el defecto tienen menos colágeno de lo normal o es de una peor calidad). Como es una proteína importante en la estructura de los huesos, causa fragilidad y debilidad de los mismos. Es precisamente esta repercusión sistémica la que determina la complejidad del manejo perioperatorio de estos pacientes. (1)

Genotípicamente presenta una gran heterogeneidad, que determina la variabilidad fenotípica. En la mayoría de casos es una enfermedad autosómica dominante debida a errores en el gen COL1A1 o COL1A2; la persona la 
padecerá si tiene una copia del gen mutada.

El trastorno también puede aparecer de novo por mutaciones esporádicas (2). Por lo general, se debe a la expresión defectuosa de las cadenas de procolágeno del tipo I. Existen muchos defectos diferentes que pueden afectar este gen y la gravedad de esta enfermedad depende del defecto específico del mismo.

El espectro de la enfermedad es sumamente amplio y abarca desde una forma mortal hasta formas leves cuya definición puede ser dudosa. La clasificación más utilizada es la de Sillence et al (1979), que los agrupa en cuatro tipos (I, II, III, IV). El diagnóstico se realiza inicialmente con las manifestaciones clínicas y las imágenes radiográficas. La densitometría ósea, la biopsia de piel y los estudios genéticos moleculares permiten un mayor acercamiento al dictamen específico. La exclusión de causas metabólicas de osteporosis es también importante como criterio basal.

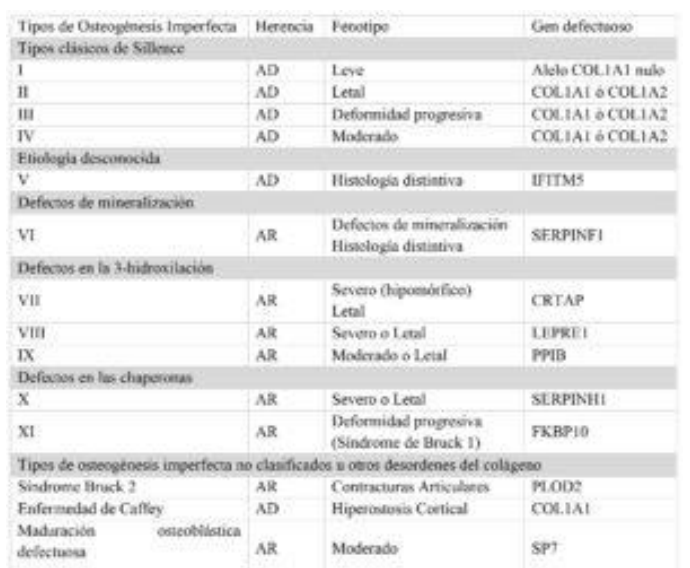

Tabla 1. Clasificación Osteogénesis Imperfecta según criterios de Scillence. (* Extraído del original)

El hallazgo aislado más importante es la fragilidad ósea, que es común a todos los tipos de OI, aunque otras manifestaciones extra-esqueléticas pueden estar presentes. Los hallazgos más comunes a cada tipo de OI se describen acordes a la clasificación de Van Dijk and Sillence (3), que se resumen en la Tabla 1.

- Osteogénesis imperfecta tipo I se asocia a baja densidad ósea. Las fracturas son raras al nacimiento, pero hay un incremento en la tasa de fracturas de huesos largos. Son comunes la presencia de escleróticas azules o grises y la aparición de sordera precoz. Deformidad de huesos largos o dentiogénesis imperfecta son infrecuentes.

- En la osteogénesis imperfecta tipo II, existe una importante afectación ósea. Ya intraútero alrededor de la semana 18-20 de gestación, se aprecian por ultrasonidos cortedad y deformidad de huesos largos y pobre osificación de huesos faciales $y$ craneales, siendo también frecuente el hallazgo de fracturas costales. La mortalidad perinatal es la norma, con alrededor del $90 \%$ de los bebés afectos en las primeras 4 semanas de vida.

- La Osteogénesis imperfecta tipo III se caracteriza por severa fragilidad ósea y progresiva deformidad esquelética. Osteopenia generalizada acompañada de fracturas son características en estudios radiográficos al nacimiento. Suelen presentar dentiogénesis imperfecta y esclerótica azul, aunque la esclera tiende a volverse menos azulada con la edad. En este tipo es frecuente hallar estatura corta y escoliosis que se inicia en la infancia y progresa con la edad. Pueden desarrollar sordera en la edad adulta. 
- Los pacientes con Ostogénesis Imperfecta tipo IV tienen fracturas recurrentes, y el grado de deformidad es desigual. La mayoría de ellos tiene escleróticas normales y es rara la alteración auditiva. Existe variabilidad incluso intrafamiliarmente, con casos de afectación media y otros con grave repercusión dentro de la misma familia.

- Finalmente, en la Osteogénesis Imperfecta tipo $\mathrm{V}$ hay calcificación progresiva de la membrana interósea y callo de fractura hiperplásico. La fragilidad ósea va de moderada a severa y no aparecen escleras azules ni dentiogénesis imperfecta.

\section{Tratamiento de la enfermedad}

El tratamiento varía en función de la edad, de la gravedad $y$ de la funcionalidad (5). En los casos leves será suficiente con algunas recomendaciones/restricciones, como la evitación de deportes de contacto, y el tratamiento ortopédico queda reservado en estos casos para manejo de fracturas. Aquellos pacientes que presenten sintomatología de moderada a severa suelen necesitar además rehabilitación, y tratamiento ortopédico de deformidades óseas de huesos largos y escoliosis.

Para aquellos con clínica severa el manejo multidisciplinar es fundamental, ya que la rehabilitación se ha demostrado beneficiosa en la funcionalidad del individuo, favoreciendo su independencia.

En cuanto al tratamiento farmacológico, la terapia con Bifosfonatos vía intravenosa ha sido durante muchos años la más usada para niños afectados de OI. Son varios los estudios que demuestran una disminución de fracturas de huesos largos y una remodelación en las fracturas vertebrales por compresión en niños, lo que repercute positivamente en el crecimiento. En el desarrollo de escoliosis en cambio, el efecto observado es pequeño. En los adultos, la terapia con bifosfonatos no muestra claros beneficios.

El mecanismo de acción es mediante producción de una reducción en la actividad osteoclástica del hueso, aumentando la densidad mineral.

La terapia intravenosa es normalmente bien tolerada. A veces puede producirse una disminución en los niveles de Calcio séricos de manera transitoria inmediatamente tras su infusión. El principal efecto adverso es la aparición en la primera infusión de un síndrome "influeza-like" caracterizado por fiebre, mialgias y náuseas.

La osteonecrosis mandibular es un conocido efecto adverso del uso de altas dosis de bifosfonatos, y nunca ha sido descrita en la Osteogénesis Imperfecta.

La hormona de crecimiento ha sido también propuesta como tratamiento (4) para paliar la deficiencia de crecimiento debido a su efecto anabolizante en el hueso. Un estudio reciente sugiere que cierto subgrupo de pacientes (mayoría OI tipo IV) podría responder a la hormona de crecimiento incrementando la altura de los mismos y con una mejora en la densidad de hueso esponjoso. Otro estudio comparó la monoterapia con bifosfonatos frente a terapia combinada bifosfonato-hormona de crecimiento, mostrando efecto sinérgico en la velocidad de crecimiento, pero sin mejoría en el riesgo de fractura. Se concluye que el uso de hormona de crecimiento parece poco eficiente en estos sujetos (4), y 
actualmente no constituye un uso clínico estándar.

\section{Revisión Bibliográfica}

La OI constituye, sin duda, una enfermedad en la que quienes la padecen se benefician de un abordaje multidisciplinar en su manejo.

Los tratamientos modernos, tanto médicos como quirúrgicos, han mejorado no sólo la calidad, sino la expectativa de vida. Dado que estos enfermos tienen alta probabilidad de sufrir múltiples fracturas que requieran intervención quirúrgica a lo largo de su vida, el manejo anestésico de los mismos merece una especial consideración.

Se realiza una revisión bibliográfica de artículos publicados en la literatura médica sobre el manejo anestésico de los sujetos con dicha afección.

Cabe resaltar que no asocian a priori mayor morbilidad cuando son sometidos a anestesia general. Tampoco mayor incidencia de vía aérea difícil ni de ingreso imprevisto en Unidad de Cuidados Intensivos (UCI).

Series de casos más antiguas, hablan de complicaciones específicas quirúrgicas o anestésicas, incluyendo hipertermia maligna, coagulopatía, fracturas yatrogénicas o presentación de Vía Aérea Difícil (VAD).

Hasta la fecha, se han publicado tres grandes revisiones de casos: Hall et al (6) en 1992 con 63 pacientes y un total de 266 intervenciones, en una revisión retrospectiva de 30 años; Bojanic (7) en 2011 con 49 casos y un total de 180 intervenciones; por último Rothschild (8) en 2018 reclutó una cohorte de 83 pacientes (entre 2008 y 2015) con un total de 205 intervenciones.
Se describen a continuación las complicaciones que históricamente se han relacionado con la Osteogénesis Imperfecta, y los hallazgos sobre su incidencia en los artículos revisados.

- Hipertermia
Intraoperatoria:

Clásicamente existe una asociación de hipertermia maligna intraoperatoria en afectos de OI; sin embargo, esto no está claramente demostrado. Algunos de estos enfermos pueden presentar aumento de la temperatura corporal sin causa conocida durante la anestesia general, que normalmente responden a medidas básicas de enfriamiento (Hall et al)

El fundamento del estudio de Bojanić fue evaluar la temperatura intraoperatoria de los sujetos con OI durante anestesia general para cirugía no cardíaca, y compararla con los controles. Los resultados mostraron que no había diferencias estadísticamente significativas tanto en el aumento de temperatura en los intervalos de medición, ni aumento significativo del endtidal de $\mathrm{CO} 2$.

En conclusión, en la práctica anestésica contemporánea los individuos con $\mathrm{OI}$ no presentan hiperpirexia u otros signos de hipermetabolismo (aumento del endtidal de $\mathrm{CO} 2$ ) cuando se comparan con otros pacientes quirúrgicos sin $\mathrm{OI}$. No se encontraron tampoco otros problemas relacionados con la anestesia, sugiriendo que prestando una atención cuidadosa, pueden someterse a una anestesia general con seguridad.

- Presentación de fracturas en miembros de forma perioperatoria

La principal característica de la OI no deja de ser la extrema fragilidad ósea que muestran debido al defecto del 
colágeno tipo 1. Oliverio describió en 1973 un caso de fractura de húmero debido al manguito de presión arterial. Muchos autores incluso recomendaban evitar la succinilcolina por miedo a que las fasciculaciones musculares produjesen fracturas, pese a que esto nunca ha sido documentado y la succinilcolina se ha usado sin eventos reseñables.

En el estudio de Bojanic no se documentó ningún caso de fractura debida manipulación.

Engel Espinosa et al (9), en un estudio retrospectivo de 29 casos y 105 intervenciones durante los años 1991 a 2009 , registraron solamente una fractura de fémur contralateral durante el traslado del paciente de la cama a la mesa de operaciones.

- Mayor incidencia de sangrado Intraoperatorio:

El colágeno defectuoso altera la interacción plaqueta-endotelio $\mathrm{y}$ produce un aumento de la fragilidad capilar, así como la afectación de la contracción de pequeños vasos después de una lesión y la alteración de la agregación plaquetaria.

También se ha visto una disminución del antígeno del factor VIII en la OI. Debido a que estos defectos se relacionan principalmente con fragilidad capilar y alteraciones plaquetarias, los estudios de coagulación preoperatorios suelen ser normales.

Los casos del estudio de E. Espinosa fueron tratados con ácido tranexámico intraoperatorio para disminuir el riesgo de hemorragia. Esto puede explicar que el número de pacientes que necesitaron una transfusión sanguínea fuera del $13,7 \%$ intraoperatoriamente y $24,1 \%$ en el postoperatorio y que no se refleje ningún caso de hemorragia importante.

En el estudio de Rothschild, hubo 35 sujetos $(17 \%)$ en los que se estimó pérdida sanguínea superior al $10 \%$. La mayoría eran OI tipo III y tipo IV, lo que sugiere asociación entre severidad de la enfermedad y riesgo de necesitar transfusión perioperatoria.

Hall en 1992 asociaba la mayor pérdida sanguínea de estos enfermos a las importantes incisiones que requerían las correcciones ortopédicas a las que eran sometidos. Sugería el uso de hipotensión controlada intraoperatoria para paliar la pérdida de volumen sanguíneo.

Edge en 1997 (10), describe un caso de hemorragia masiva en paciente con OI tipo III complicada con la aparición de CID.

- Mayor incidencia de Vía Aérea Difícil:

Los pacientes con OI son más proclives a presentar deformidades de cabeza y cuello, incluyendo macrocefalia, macroglosia, cuello corto, limitación de la movilidad cervical y deformidades mandibulares. También es importante resaltar que la gran mayoría presenta dentiogénesis imperfecta.

En los estudios revisados, la incidencia de vía aérea fallida, es nula. Sin embargo, Bojanic refiere en su estudio un aumento del uso electivo de fibrobroncoscopio (5 pacientes de 49).

En el estudio de Espinosa, no se registró ningún caso de vía aérea difícil.

Rothschild observa un 1,5\% de VAD, en las que se consigue intubación mediante glidescope o fibrobroncoscopio tras una 
laringoscopia fallida. La mayoría de estos pacientes presentaban OI tipo III.

El uso de mascarilla laríngea o intubación a través de mascarilla laríngea también se considera seguro.

- Anestesia regional y neuraxial

Sobre todo en la población obstétrica. E. Dinges (11) describe el caso de dos gemelas idénticas afectas de OI a las que se realizó cesárea con cinco meses de diferencia; la primera bajo anestesia general por presentar escoliosis severa y la segunda bajo anestesia epidural, ambas sin incidencias.

Existen series de casos con punciones neuroaxiles exitosas; si no hay deformidad anatómica importante ni alteraciones de la coagulación que la contraindiquen, la anestesia regional/neuraxial es beneficiosa en estos sujetos, en tanto en que reduce significativamente la necesidad de opioides postoperatorios.

- Otras manifestaciones extraesqueléticas.

Entre las más frecuentes, varios estudios ponen de manifiesto la existencia de valvulopatías cardíacas, siendo la insuficiencia aórtica la más frecuente. El estudio de Bonilla et al (12), de casos y controles, compara hallazgos ecocardiográficos en enfermos con OI y en la población general, no encontrando diferencias significativas en el hallazgo de valvulopatías. También se encontró que la raíz valvular aórtica en relación a la superficie corporal era ligeramente mayor en OI. También se han descrito casos de disección aórtica en estos sujetos.

En cuanto a las manifestaciones del SNC se incluyen la luxación atloaxoidea y la hidrocefalia como las más frecuentes.
Por último, debemos tener presente que al ser individuos con múltiples intervenciones quirúrgicas, el desarrollo de alergia al látex debe ser tenido en cuenta y programarlos como tal.

\section{Desarrollo Caso Clínico}

Se procede a continuación a la descripción de un caso clínico en nuestro centro (Hospital Rehabilitación y Traumatología Virgen del Rocío Sevilla).

Se trata de una paciente mujer, de 37 años de edad, ASA II, afecta de OI tipo III. Presenta caída de silla de ruedas autopropulsable con fractura de fémur proximal (Imagen 1). Se realiza intervención quirúrgica programada para reducción y osteosíntesis de la misma.

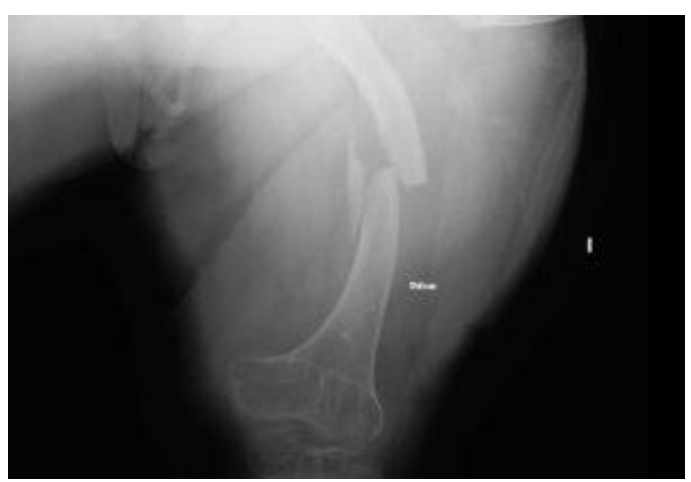

Imagen 1. Imagen radiográfica de la fractura en Urgencias. Imagen original de autor.

El estudio preanestésico no mostraba alergias reconocidas a medicamentos y sustancias.

Como antecedentes destacables, era fumadora de unos 20 cigarrillos/día, presentaba Osteogénesis Imperfecta tipo III, con múltiples fracturas previas en miembros, tratadas de forma conservadora. No realizaba tratamiento domiciliario de ningún tipo.

Exploración de la Vía aérea: Mallampati 2. Test de la mordida 1. Dentición sin alteraciones, salvo algunas piezas careadas. 
Respecto a las pruebas complementarias:

Analítica:

Hemoglobina 13,2g/dL; Plaquetas: $273000 \mathrm{~mm}^{3}$; TPTA $24 \mathrm{seg}$. INR 0,98. Fibrinógeno 5,1g. Glucemia 149 g/dL, $\mathrm{Cr}$ 0,53. Iones en rango. El ECG mostraba ritmo sinusal a 75 latidos por minuto, sin alteraciones de la repolarización.

Ya en quirófano, se realizó monitorización hemodinámica mediante presión arterial no invasiva, saturación de Oxígeno y electrocardiograma. Se estimó el riesgo hemorrágico como moderado, y se decidió anestesia general por importante escoliosis y ansiedad de la paciente.

La inducción transcurrió sin incidencias, se comprobó correcta ventilación manual (escala de Han I/IV). Intubación orotraqueal mediante videolaringoscopio al primer intento con tubo endotaquel

flexometálico $\mathrm{n}^{\circ} 7,5$.

El mantenimiento se realizó mediante anestesia general balanceada con Desfluorane a 0,8-0,9 CAM.

Se canalizó arteria radial derecha para monitorización de Presión Arterial Invasiva, sondaje vesical y control de la temperatura.

Durante la intervención, la paciente se mantuvo estable hemodinámicamente, manteniendo tensión arterial $\mathrm{y}$ frecuencia cardíaca en torno a $+/-20 \%$ de los valores de referencia. $\mathrm{La}$ temperatura osciló durante la intervención $36^{\circ}-36,5^{\circ} \mathrm{C}$.

Como fluidoterapia se emplearon un total de $750 \mathrm{ml}$ cristaloides. No fue necesaria la administración de hemoderivados.

Se realizó reducción cerrada de la fractura mediante control radiológico y osteosíntesis percutánea con placa lateral derecha.

Tras finalizar la intervención, la paciente fue extubada en quirófano sin incidencias tras reversión de bloqueo neuromuscular. Se trasladó a la Unidad de Reanimación Postanestésica para continuidad de cuidados, donde permaneció tres horas tras las cuales se trasladó a planta de hospitalización con aceptable control del dolor y sin signos de sangrado activo.

A los tres días de la intervención, dada la evolución favorable (Imagen 2) y la ausencia de complicaciones, fue dada de alta a su domicilio.

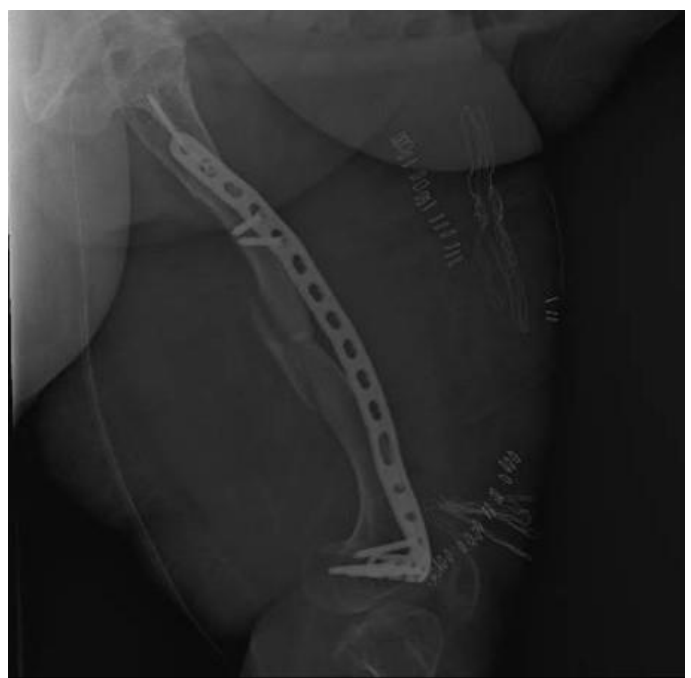

Imagen 2. Control radiográfico tras reducción y osteosíntesis. Imagen original de autor.

\section{Conclusiones}

Los pacientes afectos de Osteogénesis Imperfecta son subsidiarios de pasar a lo largo de su vida en más de una ocasión por quirófano, sea para correcciones escolióticas o bien para tratamiento de fracturas.

El médico anestesiólogo tiene el importante papel de realizar una correcta historia clínica en busca de las compilaciones más frecuentemente asociadas a esta entidad, y de realizar una buena exploración de la vía aérea 
con el fin de prever dificultades en la intubación.

Con ello, actualmente puede considerarse que estas intervenciones pueden ser llevadas a cabo mediante anestesia general o locorregional de manera segura para los pacientes.

\section{Bibliografía}

(1) Palomo T, Vilaça T, Lazaretti-Castro M. Osteogenesis imperfecta: diagnosis and treatment. Curr Opin Endocrinol Diabetes Obes. 2017 Dec;24(6):381-388. DOI: $\underline{10.1097 / M E D .0000000000000367}$

(2) Palenzuela-Ramos Y, Moreira-Díaz LR, Padrón-Álvarez JE. Osteogénesis imperfecta, reporte de un caso. Universidad Médica Pinareña. 2020;16(2):1-6.

(3) Sillence DO, Senn A, DanksDm. Genetic heteroeneity in osteogenesis imperfecta. J Med Genet. $\quad 1979 ; \quad 16$ : $101-116$. DOI: $\underline{10.1136 / \mathrm{jmg} \cdot 16.2 .101}$

(4) Rossi, Vittoriaa; Lee, Brendana,b; Marom, Ronita,b Osteogenesis imperfecta: advancements in genetics and treatment, Current Opinion in Pediatrics: December 2019 Volume 31 - Issue 6 - p 708-715. DOI: $10.1097 / \mathrm{MOP} .0000000000000813$

(5) Trejo P, rauch F. Osteogenesis Imperfecta in children and adolescents- new developments in diagnosis and treatment. Osteoporos Int 2016; 27: 3427-3437. (PubMed)

(6) Hall RMO, Henning RD, Trejo P, rauch F. Osteogenesis Imperfecta in children and adolescents- new developments in diagnosis and treatment. Osteoporos Int 2016; 27: 34273437Brown TCK, Cole WG. Anaesthesia for chidren with Osteogenesis Imerfecta- a review covering 30 years and 266 anaesthetics. Paediatr. Anaesth. 1992; 2:1 15-21.

(7) Bojanić K, Kivela JE, Gurrieri C, Deutsch E, Flick R, Sprung J, Weingarten TN. Perioperative course and intraoperative temperatures in patients with osteogenesis imperfecta. Eur J Anaesthesiol. 2011
May;28(5):370-5.

DOI:

10.1097/eja.0b013e3283459616 (ubMed)

(8) Rothschild L, Goeller JK, Voronov P, Barabanova A, Smith P. Anesthesia in children with osteogenesis imperfecta: Retrospective chart review of 83 patients and 205 anesthetics over 7 years. Paediatr Anaesth. 2018 Nov;28(11):1050-

1058. DOI: $10.1111 /$ pan. 13504

(9) Engel Espinosa W, Arrázola Cabrera B, Peralta Rodríguez P, Fernández Izquierdo MC, García Molina C, Ortigosa Solórzano E. Experiencia en el tratamiento anestésico de los pacientes afectos de osteogénesis imperfecta [Anesthetic treatment of patients with osteogenesis imperfecta]. Rev Esp Anestesiol Reanim. 2011 Mar;58(3):151-5.

(10) Edge G, Okafor B, Fennelly ME, Ransford AO. An unusual manifestation of bleeding diathesis in a patient with osteogenesis imperfecta. Eur J Anaesthesiol. 1997 Mar;14(2):215-9. doi: 10.1046/j.13652346.1997.00115.x. $\quad$ PMID: 9088824. DOI: $10.1046 / \mathrm{j} .1365-2346.1997 .00115 . \mathrm{x}$

(11) Dinges E, Ortner C, Bollag L, Davies J, Landau R. Osteogenesis imperfecta: cesarean deliveries in identical twins. Int J Obstet Anesth. 2015 Feb;24(1):64-8. doi: 10.1016/j.ijoa.2014.07.006. Epub 2014 Jul 30. PMID: 25433579. (PubMed)

(12) Bonilla Jiménez V, Saavedra Felero J, Alberca Vela MT, et al. Alteraciones cardíacas en la osteogénesis imperfecta. Estudio ecocardiográfico de casos y controles. Med Clin (Barc), 2010; 135: 681-684 (HTML)

(*) Valadares ER, Carneiro TB, Santos PM, Oliveira AC, Zabel B. What is new in genetics and osteogenesis imperfecta classificatio

Correspondencia al autor

María Dolores Serrano de los Santos marserdel@gmail.com delia0407@hotmail.com

FEA Anestesiología y Reanimación Hospital de Rehabilitación y Traumatología

H. U. Virgen del Rocio Sevilla.

Aceptado para el blog en marzo de 2021 